\title{
CD36 in Atherosclerotic Coronary Artery Disease: Correlation with Angiogenesis and Inflammation
}

\author{
Enas A. El-Hussieny ${ }^{1}$, Amira M. Gamal Eldeen ${ }^{2,3}$, Roba M. Talaat ${ }^{4}$, Ahmed F. Tamara $^{5}$, \\ Mohamed S. Salama ${ }^{6}$ and Nadia M. El-Beih ${ }^{1}$ \\ ${ }^{1}$ Department of Zoology, Faculty of Science, Ain Shams University, Cairo, Egypt \\ ${ }^{2}$ Department of Cancer Biology Laboratory, Center of Excellence for Advanced Sciences, National Research Center (NRC), Dokki, 12622, Cairo, Egypt \\ ${ }^{3}$ Department of Biochemistry, National Research Center, Dokki, 12622, Cairo, Egypt \\ ${ }^{4}$ Department of Molecular Biology, Genetic Engineering and Biotechnology Research Institute (GEBRI), University of Sadat City, Egypt \\ ${ }^{5}$ Department of Cardiology, Faculty of Medicine, Ain Shams University, Cairo, Egypt \\ ${ }^{6}$ Department of Entomology, Faculty of Science, Ain Shams University, Cairo, Egypt
}

Received: December 19, 2014; Accepted: April 06, 2015; Published: April 15, 2015

*Corresponding author: Roba M. Talaat, Department of Molecular Biology, Genetic Engineering and Biotechnology Research Institute (GEBRI), University of Sadat City, Egypt, E- mail: robamtalaat@yahoo.com

\begin{abstract}
Background: CD36 is one of the macrophage scavenger receptor that has been implicated as a key player in the pathogenesis of atherosclerosis.
\end{abstract}

Aim: This study focused on the role of CD36 in coronary artery disease (CAD) and its association with proangiogenic and inflammatory mediators.

Patients and Methods: We studied 100 atherosclerotic CAD patients and 100 healthy controls. All patients had angiographic evidence of stenosis $\geq 50 \%$ in at least one major coronary artery Soluble CD36 (sCD36), peripheral blood mononuclear cells (PBMCcCD36), vascular endothelial and platelet- derived growth factors (VEGF \& PDGF), Tumor necrosis factor (TNF- $\alpha$ ) and cycloxygenase-2 (COX-2) were measured using enzyme-linked immunosorbent assay (ELISA). CD36 mRNA expression was measured using reverse transcriptase polymerase chain reaction (RT-PCR).

Results: A significant reduction in cCD36 expression (protein and mRNA) and plasma levels of sCD36, VEGF, PDGF in CAD patients was demonstrated. In contrast, a marked elevation in the plasma level of TNF- $\alpha$ was observed. There was a strong inverse correlation between severity of CAD and SCD36 $(r=-0.413 ; P<0.001), \operatorname{cCD} 36(r=-0.4663$; $P<0.001)$, CD36 mRNA $(r=-0.328 ; P<0.01)$. Furthermore, CD36 was found to be positively correlated with VEGF $(r=0.446 ; P<0.001)$ and PDGF $(r=0.287 ; P<0.05)$, while it was negatively correlated with TNF- $\alpha(r=-0.352 ; P<0.01)$.

Conclusion: CD36 deficiency might represent a good expression marker for the development of CAD in Egyptian patients in association with inflammatory and proangiogenic mediators. Targeting the underling mechanism of the direct and inverse association of CD36 with angiogenic and inflammatory mediators could be used in the treatment of athero-inflammatory disorders.

Keywords: Coronary artery disease; CD36; Angiogenesis; Inflammation.

\section{Abbreviations}

CAD: Coronary Artery Disease; VEGF: Vascular Endothelial Growth Factor; PDGF: Platelet - Derived Growth Factor; TNF- $\alpha$ : Tumor Necrosis Factor- $\alpha$; COX- 2: Cycloxygenase-2; HC: Hypercholesterolemia; HT: Hypertension; DM: Diabetes Mellitus; RT-PCR: Reverse Transcription Polymerase Chain Reaction.

\section{Introduction}

Cardiovascular Diseases (CVDs) accounting for $\sim 38 \%$ of all deaths worldwide. Without doubt, the largest contributor to CVD is Coronary Artery Disease (CAD), the leading cause of the death in developed countries [1]. By 2015, almost 20 million people will die from CVDs [2]. In Egypt CVDs are now the main causes of death among Egyptians. In 1970, CVDs were accounted for $12.4 \%$ of all deaths, whereas two decades later they were responsible for $42.5 \%$ of the mortality [3]. Major risk factors of CVDs include Hypercholesterolemia (HC), Hypertension (HT), Diabetes Mellitus (DM) and smoking [4].

Atherosclerosis, the main cause of CAD, is a progressive and chronic inflammatory disease in which lipids, immune cells, vascular smooth muscle cells, and extracellular matrix accumulate in the subendothelial space to form the growing atherosclerotic lesion. The risk for of cardiovascular events is related to the composition and stability of the plaque rather than to the degree of arterial stenosis, and many studies suggest that inflammation is a critical determinant of plaque stability [5]. More than $80 \%$ of sudden cardiac death is caused by atherosclerosis [6]. The progression of the atherosclerotic disease and the increasing severity of atherosclerosis relate not only to the presence and extent of cardiovascular risk factors but also to the persistence of risk factors over time [4]. The study by Allah, et al. [7] found that atherosclerotic carotid artery disease (intima-media thickness and/ or plaques) was present in $41 \%$ of the study population. 
CD36 is one of the monocyte-derived macrophage scavenger receptors that have been implicated as a key player in the pathogenesis of atherosclerosis [8]. It is believed to play a critical role in the initiation and progression of atherosclerosis through its ability to bind and internalize modified lowdensity lipoprotein, facilitating the formation of lipid-engorged macrophage foam cells [9]. A variety of studies have been shown that CD36 deficiency may be related to the metabolic syndrome, which is strongly associated with atherosclerotic CVD [10].

Inadequate blood supply to the heart and other tissues resulting from partially lose of the functional blood flow and insufficient new blood vessel growth is a hallmark feature of atherosclerosis. The formation of new blood vessels out of preexisting capillaries (angiogenesis) is a consequence of events that is important in atherosclerosis. The most potent pro angiogenic factors are Vascular Endothelial Growth Factor (VEGF) and platelet- derived growth factor (PDGF). They played a significant role in angiogenesis and regulation of vascular endothelial cell growth and maintenance and development of new blood vessels [11].

In atherosclerotic plaques, monocytes/macrophages are significant producer of inflammatory cytokines that have a central role in atherogenesis, and cause the clinical manifestations and acute clinical complications of atherosclerosis [12]. Tumor necrosis factor (TNF- $\alpha$ ) is involved in the pathogenesis of atherosclerosis and it can initiate many signaling pathways resulting in production of factors influencing angiogenesis [13]. Substantial evidence indicates that unregulated cycloxygenase-2 (COX-2) expression and prostaglandin synthesis influence chronic inflammatory condition, including atherosclerosis and its complications. COX-2 is rapidly induced by various stimuli, including proinflammatory cytokines, such as TNF- $\alpha$, growth factors, resulting in prostaglandin synthesis associated with inflammation [14].

Risk factors for the development of atherosclerotic CAD have been identified, but several biomarkers may also be important in identifying patients at risk. Therefore, the aim of this study was to evaluate the potential predictive role of CD36 expression in atherosclerosis severity in Egyptian patients. The study was also focused on the relationship between the inflammatory (TNF- $\alpha$, and COX-2), angiogenic (VEGF and PDGF) mediators and the scavenger receptor (CD36) in the development of accelerated atherosclerotic CAD in Egyptian patients. To the best of our knowledge, this study is the first to be done in Egyptian patients with CAD

\section{Patients and Methods}

\section{Study population}

The study population was consisted of 100 consecutive atherosclerotic patients (69 males and 31 females) with mean age $54 \pm 7$ years (range 35-77 years), who had been referred to Nasser Institute Hospital, Egypt International Hospital, Specialized Hospital of Ain Shams Universities, El-Demerdash Hospital and Cardiovascular Cath-Lab Center, Egypt. All investigations were done in accordance with the Ministry of Health, Health and Human Ethical Clearance Committee guidelines for Clinical Researches. Ain-Shams University local Ethics Committee approved the study protocol. All patients and healthy controls agreed to be enrolled in this study and written consent was obtained from all participants.

All patients had angiographic evidence of stenosis $\geq 50 \%$ in at least one major coronary artery or their main branches. Stenosis after a careful history was taken; the patients underwent a physical examination, chest X-ray, 12-lead Echocardiography (ECG) was performed to detect segmental wall motion abnormalities (SWMA) as a result of myocardial ischemia, and ultrasound examination of the heart. Blood was drawn from fasting patients on the morning of catheterization for assay of glucose and lipid concentrations by standard laboratory methods. Risk factors for cardiovascular disease, carotid artery ultra sonography, and coronary artery angiography-left ventriculography were performed. According to the number of diseased vessels, the patients were classified into three groups: Patients with one- (SV), two- (DV), or Multiple-Vessels Disease (MV). Clinical features of CAD cases and controls were shown in Table 1.

The study was also included 100 normal healthy individuals to serve as a control group, including 25 females and 75 males.

Table 1: Demographics and clinical status of Egyptian CAD patients.

\begin{tabular}{|c|c|}
\hline Characteristic & Patients (\%) $N=100$ \\
\hline Age $(y r)(m e a n \pm S E)$ & $58 \pm 10$ \\
\hline \multicolumn{2}{|c|}{ Gender (\%) } \\
\hline Male & $69 \%$ \\
\hline Females & $31 \%$ \\
\hline \multicolumn{2}{|c|}{ Coronary risk factors } \\
\hline Family history & $25.60 \%$ \\
\hline Diabetes Mellitus (DM) & $13.30 \%$ \\
\hline Hypertension (HT) & $29.30 \%$ \\
\hline Hypercholesterolemia (HC) & $13.30 \%$ \\
\hline $\mathrm{DM}+\mathrm{HT}$ & $21.20 \%$ \\
\hline $\mathrm{DM}+\mathrm{HC}$ & $8 \%$ \\
\hline $\mathrm{HT}+\mathrm{HC}$ & $24 \%$ \\
\hline $\mathrm{DM}+\mathrm{HT}+\mathrm{HC}$ & $45.30 \%$ \\
\hline \multicolumn{2}{|c|}{ Coronary angiography } \\
\hline Severe & $60 \%$ \\
\hline Moderate & $30 \%$ \\
\hline Mild & $10 \%$ \\
\hline \multicolumn{2}{|c|}{ Number of stenosed vessels } \\
\hline Single vessel (1) & $38.30 \%$ \\
\hline Double vessels (2) & $31.60 \%$ \\
\hline Multi-vessels ( 3 or more) & $28.30 \%$ \\
\hline \multicolumn{2}{|c|}{ Smoking status } \\
\hline Mild & $10 \%$ \\
\hline Moderate & $11.60 \%$ \\
\hline Severe & $30 \%$ \\
\hline
\end{tabular}


Controls included persons who had no major risk factors such as family history of early cardiovascular disease, diabetes, hypertension, hypercholesterolemia, and smoking. They had normal resting ECG or with no blood vessel stenosis or disease (at angiography). Blood samples were drawn from patients prior to angiography. Plasma were separated by centrifugation at $1500 \mathrm{rpm}$ for $15 \mathrm{~min}$ at $4^{\circ} \mathrm{C}$, aliquotted, and stored at $-80^{\circ} \mathrm{C}$ until analysis. The Peripheral Blood Mononucleated Cells (PBMCs) were obtained from heparinized blood by centrifugation through a Ficoll-Hypaque separating media (Bio Basic Canada Inc).

\section{Evaluation of risk factor variables}

The recorded risk factors included smoking status, HC, DM, HT and family history of CAD. Current smokers were categorized according to the number of cigarette smoked per day as reported at baseline into mild smoker (1-10 cigarette/ day), moderate (1020 cigarette/ day), and severe smokers (20 or more cigarette/ day). Non-smokers group had never smoked any cigarette. Hypertension was defined as blood pressure over 140/90 mm Hg, as measured on several occasions, or the use of antihypertensive treatment; DM as a fasting plasma glucose over $126 \mathrm{mg} / \mathrm{dl}$ or the use of glucose-lowering treatment; hyperlipidemia as low density lipoprotein levels over $130 \mathrm{mg} / \mathrm{dl}$ or high density lipoprotein $<35 \mathrm{mg} / \mathrm{dl}$ and triglycerides $>200 \mathrm{mg} / \mathrm{dl}$ or the use of lipid-lowering therapy. Accordingly, our CAD patients were sub-classified based on the presence of one or more risk factors into: DM, HT, HC, DM+HC, DM+HT, HT+HC, and DM+HT+HC.

\section{Estimation of CD36}

Soluble and PBMCs CD36 (sCD36 and cCD36) were quantified by indirect ELISA as previously described [15]. Plasma CD36 levels were expressed as milli absorpance $(\mathrm{mA})$.

\section{Analysis of CD36 mRNA expression}

Expression of CD36 by PBMCs was performed using reverse transcription polymerase chain reaction (RT-PCR). Total RNA was isolated from PBMCs samples using BIOZOL reagent (Bioflux) following manufacturer's instructions. Purity and concentration of total RNA was quantified using UV-microplate reader (FLUOstar OPTIMA), on the basis of the A260/ A280 ratio. RT-PCR was performed using total RNA template, where $1 \mu \mathrm{g}$ of total RNA was reversely transcribed to produce cDNA by sequential incubation with anchored $0.6 \mu \mathrm{m}$ oligo-dT primer and RT enzyme (SibEnzyme, Ltd, Russia) at $37^{\circ} \mathrm{C}$ for $10 \mathrm{~min}$, $42^{\circ} \mathrm{C}$ for $1 \mathrm{~h}$ and $70^{\circ} \mathrm{C}$ for $10 \mathrm{~min}$. The final volume for each PCR reaction was $25 \mu$ that was consisted of DreamTaq Green PCR Master Mix (2X) (Fermentas, Thermo Fisher Scientific Inc., USA), 50 pmoles of specific primers for human CD36: Sense: 5'-TCCTCGAAGAAGGTACAATTGC-3' and antisense: 5'-5'-CAATACAATGACATTTGCCAAG-3'. PCR cycling conditions were consisted of $95^{\circ} \mathrm{C}$ for 2 min followed by 40 cycles $\left(94^{\circ} \mathrm{C}\right.$ for 40 seconds, $60^{\circ} \mathrm{C}$ for 60 seconds and $72^{\circ} \mathrm{C}$ for 60 seconds) and then a final extension cycle at $72^{\circ} \mathrm{C}$ for $10 \mathrm{~min}$ [16]. PCR products (250 bp) were visualized by $2.5 \%$ agarose gel electrophoresis. The sizes of PCR products were determined relatively to the migration of a 50 bp step ladder (Fermentas). The signed intensity of the bands was quantified by UV gel documentation system (Biometra ${ }^{\circledR}$ goettingen, Germany, Bioanalyze 1.0).

\section{Estimation of plasma pro-angiogenic factors}

Plasma levels of VEGF, PDGF and TNF- $\alpha$ were quantified by ELISA as previously described [16], using matched paired antibodies (R\&D Systems). The ELISA reader-controlling software (Softmax) readily processes the digital data of raw absorbance values into a standard curve from which unknown concentrations can be derived directly. Results were expressed as $\mathrm{pg} / \mathrm{ml}$ of plasma.

\section{Estimation of COX-2 by Dot Blot assay}

Plasma COX-2 was quantified by Dot Blot assay. A polyvinylidene difluoride (PVDF) membrane (Amersham Pharmacia Inc., CA, USA) was pre-wet in 100\% methanol for $2 \mathrm{~min}$, and then soaked in Tris-buffered saline (TBS) (50 mM Tris- $\mathrm{HCl}$ and $150 \mathrm{mM} \mathrm{NaCl}$; pH 7.5) for rehydration. $50 \mu \mathrm{l}$ of plasma were added to each well, and incubated for $45 \mathrm{~min}$. After washing with TTBS (TBS containing Tween 20), $200 \mu \mathrm{l}$ of blocking buffer (TTBS with 7\% FBS) were added for 60 min. Goat anti-human COX-2 (diluted 1:1000; Abcam, MA, USA) and rabbit anti-goat polyclonal antibody- horseradish-peroxidase conjugated (diluted 1:1000; Sigma) were used. After $60 \mathrm{~min}$ incubation, the membrane was washed with TBS then soaked in 3,3' Diaminobenzidine (DAB) solution (5 mg DAB + $10 \mathrm{ml}$ TTBS $+30 \% \mathrm{H}_{2} \mathrm{O}_{2}$ ) (Biobasic). After color development, the membrane was soaked in distilled water, dried for imaging and analyzed by BioDocAnalyze (BDA) soft ware (Biometra $\left.{ }^{\circledR}\right)$.

\section{Statistical analysis}

All of the statistical analysis was performed using the Statistical Package for Social Science (SPSS) version 10. Data are presented as means with corresponding standard error. Comparisons among different groups of patients were performed by one-way analysis of variance (ANOVA). Tukey's post-hoc test and Dunnett's test for multiple comparisons were used. Correlation between variables was determined using Spearman's correlation test. In all of the tests the level of significance was set at $P<0.05$. For those variables that were significantly influencing serum CD36 levels $(P<0.05)$, a standard linear multiple regression analysis with CD36 levels as the dependent variable was performed. For the regression model, adjusted $\mathrm{R}^{2}, \beta, \mathrm{b}$-value and standard error b were recorded.

\section{Results}

\section{Soluble CD36, PMNCs CD36, and Expression of CD36 mRNA in PMNCs in CAD patients versus control subjects}

sCD36 level in plasma and cCD36 expression (cCD36 and CD36 mRNA) of CAD patients classified according to risk factors is presented in Figures 1 \& 2. sCD36 level and cCD36 expression were significantly reduced in all groups of CAD patients suffered from risk factors [DM $(P<0.05, P<0.001)$, HT $(P<0.001, P<0.05)$, HC $(P<0.001, P<0.01)$, DM+HT $(P<0.001, P<0.001), \mathrm{DM}+\mathrm{HC}$ $(P<0.05, P<0.01)$, HT+HC $(P<0.01, P<0.001)$ and $\mathrm{DM}+\mathrm{HT}+\mathrm{HC}$ 

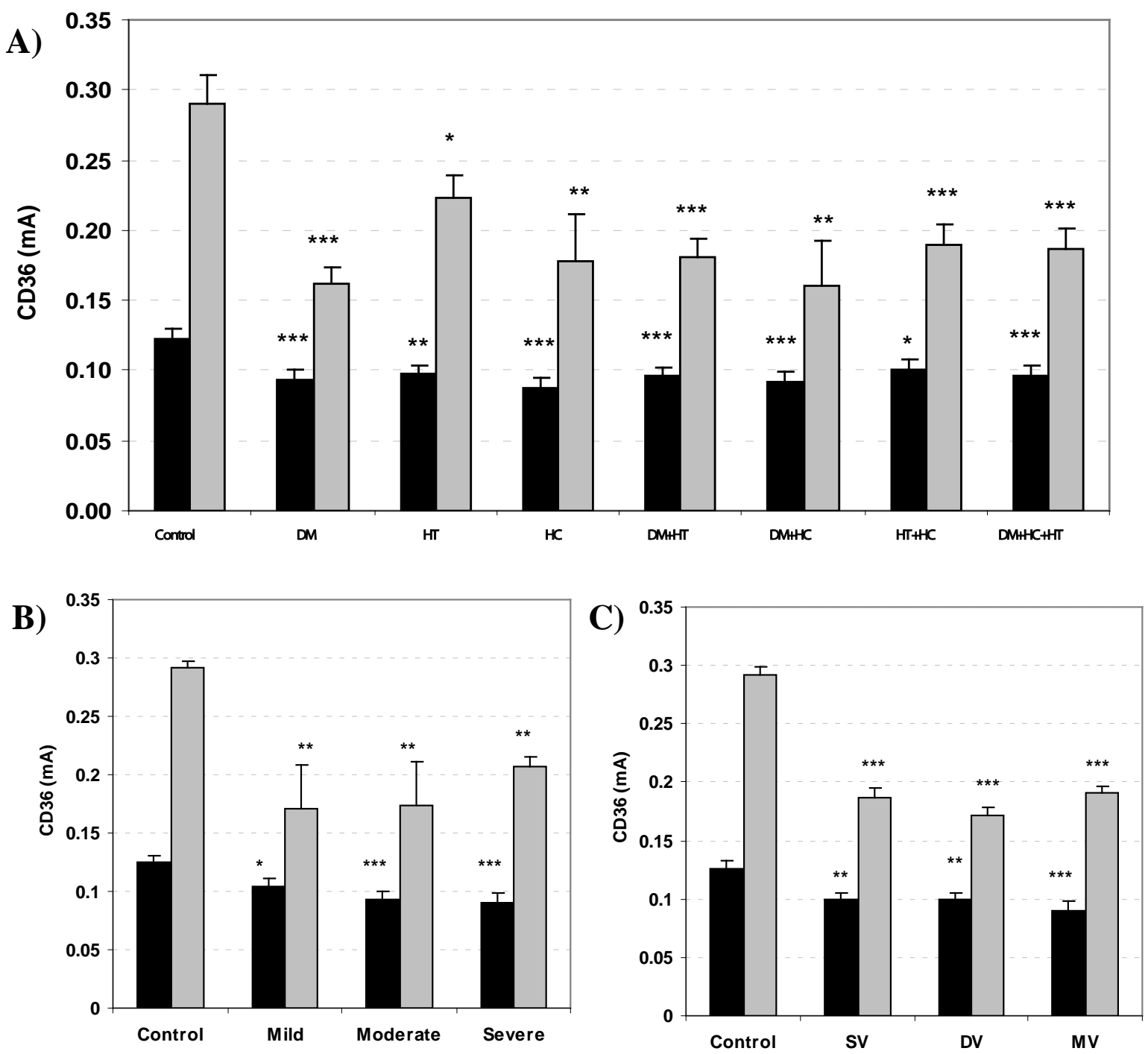

Figure 1: Plasma (black bars) and PMNCs surface (grey bars) levels of CD36 in CAD patients classified according to risk factors (a), smoking status (b), and number of stenoic arteries (c). Results are expressed as mean \pm standard error. ${ }^{*}$ Denotes groups significantly different from controls. ${ }^{*} P<$ $0.05,{ }^{* *} P<0.01,{ }^{* * *} P<0.001$.

DM: Diabetes Mellitus; HT: Hypertension; HC: Hypercholesterolemia; SV: Single Vessel Stenosis; DV: Double Vessel Stenosis; MV: Multi-Vessel Stenosis

$(P<0.001, P<0.001)]$, as shown in figure 1a, where's a reduced cCD36 mRNA expression was noticed in hypertensive groups of CAD patients $[\mathrm{HT}(P<0.05)$, DM+HT $(P<0.01)$ and $\mathrm{DM}+\mathrm{HT}+\mathrm{HC}$ $(P<0.01)]$ in comparison to the healthy control (Figure 2c). When considering smoking status groups, a significant reduction in sCD36 level $(P<0.05$ and $P<0.001)$ and cCD36 expression $(P<0.01)$ were demonstrated in all groups of different smoking degrees in relation to normal subjects (figure1b). cCD36 mRNA expression was significantly reduced $(P<0.01)$ only in severe smokers versus control subjects (figure $2 \mathrm{~b}$ ). The analysis according to the number of stenotic arteries indicated that SV, DV and MV groups had a significant reduction in sCD36 level ( $P$ $<0.001, P<0.01, P<0.001$ for SV, DV and MV; respectively) and cCD36 expression $(P<0.001)$ versus control subjects (figure $1 \mathrm{c}$ ). A significant reduction in mRNA expression of cCD36 was found in SV $(P<0.05)$, DV $(P<0.01)$ and MV $(P<0.01)$ when compared to the healthy controls (Figure 2d).

\section{Evaluation of plasma VEGF plasma PDGF}

Compared with normal controls, a significant reduction in VEGF was observed in CAD patients who suffered from hypercholesterolemia [HC $(P<0.05)]$ and hypertensive groups $[\mathrm{DM}+\mathrm{HT}(\mathrm{P}<0.001)$; HT+HC $(P<0.01)$ and DM+HT+HC $(P<$ $0.001)]$, as shown in figure 3 a. It was noticed that there was a significant decrease in plasma PDGF concentration in diabetic $(P$ $<0.001)$, hypercholesterolemic patient $(P<0.01)$ and the patients suffered from combined DM+HT, HT+HC and DM+HC $(P<0.001)$ as shown in figure $3 \mathrm{a}$.

Our findings revealed that there was a significant reduction in plasma VEGF in mild, moderate $(P<0.05)$ and severe smokers $(P$ 

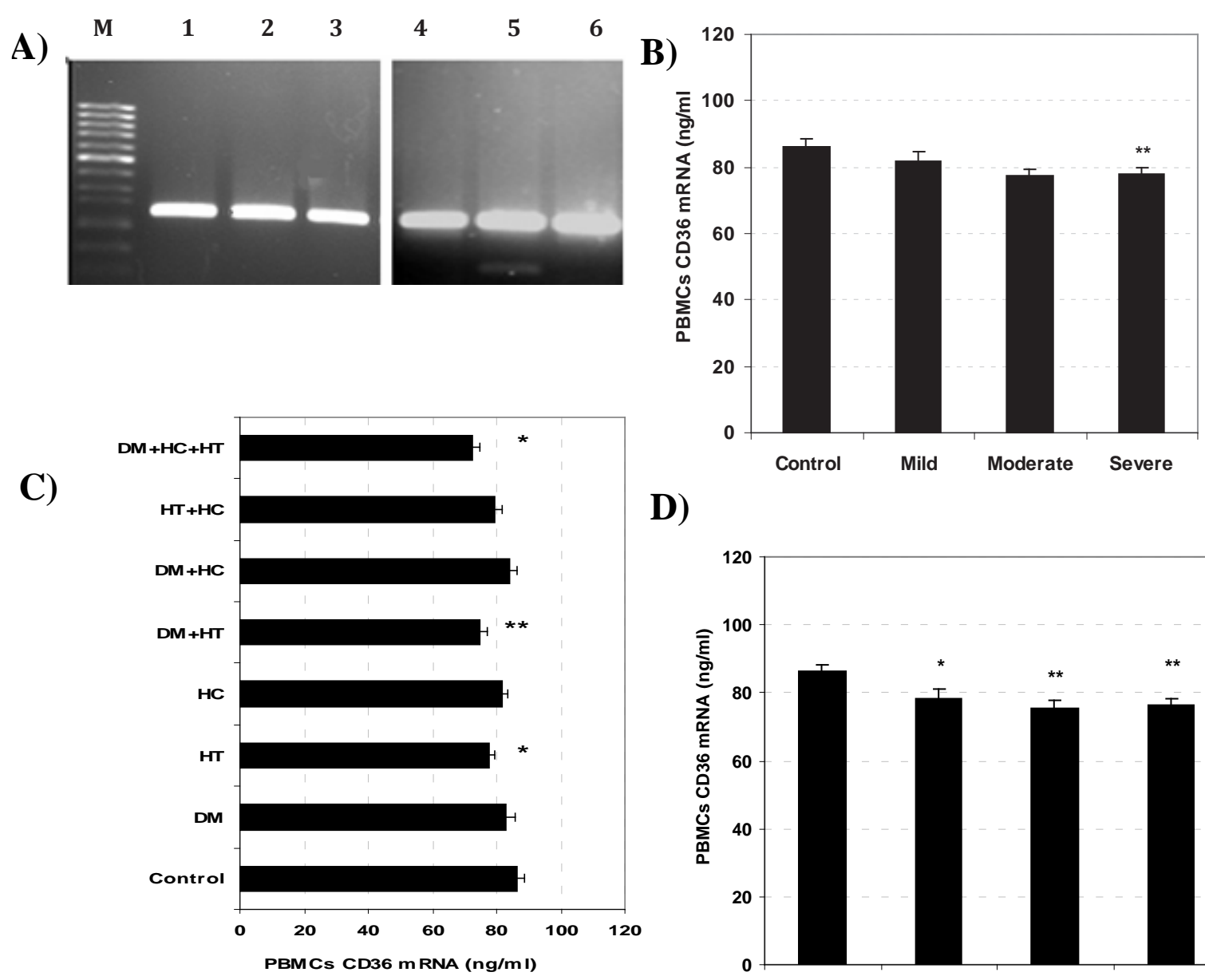

D)

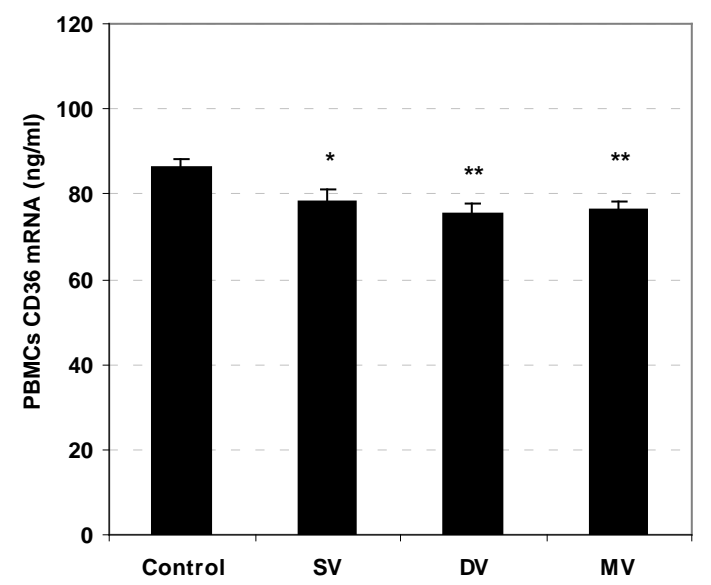

Figure 2: CD36 mRNA expression quantified by RT-PCR. (a) 2\% agarose gel for the amplified fragment (250 bp) Lane 1: DNA ladder (50 bp); lane (2-4): CD36 of CAD patients; lane (5-7): CD36 of healthy subjects. CD36 mRNA in CAD patients classified according to according to risk factors (b), smoking status (c), and number of stenoic arteries (d). Results are expressed as mean \pm standard error. *Denotes groups significantly different from controls ${ }^{*} P<0.05,{ }^{* *} P<0.01$.

DM: Diabetes Mellitus; HT: Hypertension; HC: Hypercholesterolemia; SV: Single Vessel Stenosis; DV: Double Vessel Stenosis; MV: Multi-Vessel Stenosis.

$<0.01$ ) compared to the healthy controls (figure 3b). Additionally, a significant reduction $(P<0.001)$ was recorded in plasma PDGF in mild, moderate, and severe smokers (figure $3 \mathrm{~b}$ ). Concerning stenosis, diminution in plasma VEGF and PDGF levels was also demonstrated in CAD patients with SV, DV and MV stenosis compared to the healthy controls $(P<0.001)$ (figure $3 \mathrm{c}$ and $3 \mathrm{c}$ ).

\section{Evaluation of plasma TNF- $\alpha$ and COX-2}

In comparison to the healthy controls, patients had a significant increase in plasma TNF- $\alpha$ secretion level in DM $(P$ $<0.05)$ and HT $(P<0.001)$. CAD patients who suffered from $\mathrm{HC}$ had insignificant reduction in TNF- $\alpha$ level. In groups of combined risk factors, $\mathrm{DM}+\mathrm{HT}, \mathrm{DM}+\mathrm{HC}$, and $\mathrm{HT}+\mathrm{HC}$ groups have a significant increase $(P<0.01)$ in TNF- $\alpha$ level. A significant $(P$ $<0.001$ ) elevation in plasma TNF- $\alpha$ level was demonstrated in $\mathrm{DM}+\mathrm{HT}+\mathrm{HC}$ group (figure $4 \mathrm{a}$ ). Degrees of smoking frequency showed a significant increase in plasma TNF- $\alpha$ in mild $(P<0.001)$, moderate and severe smoking $(P<0.01)$ as compared to the healthy controls. However, compared with mild smoking status, a significant reduction in TNF- $\alpha$ was observed in moderate $(P$ $<0.05)$ and severe $(P<0.05)$ smokers (figure $4 \mathrm{~b})$. According to number of stenotic arteries, plasma TNF- $\alpha$ showed a significant increase in SV $(P<0.001)$, DV $(P<0.05)$ and MV $(P<0.001)$ stenosis groups, as compared to the healthy controls (figure $4 \mathrm{c}$ ). The maximum production was observed in MV patients. There was a non-significant change $(P>0.05)$ in plasma COX-2 levels in CAD patients classified according to risk factors, smoking status or stenosis, as compared to the healthy controls.

\section{Statistical correlations}

CAD progression in Egyptian patients showed statistically significant direct correlation with plasma levels of TNF- $\alpha(r=$ $0.480, P<0.001)$ and indirect significant correlation with VEGF $(r$ $=-0.499, P<0.001), \operatorname{sCD} 36(r=-0.413, P<0.001), \operatorname{cCD} 36(r=-0.466$, 

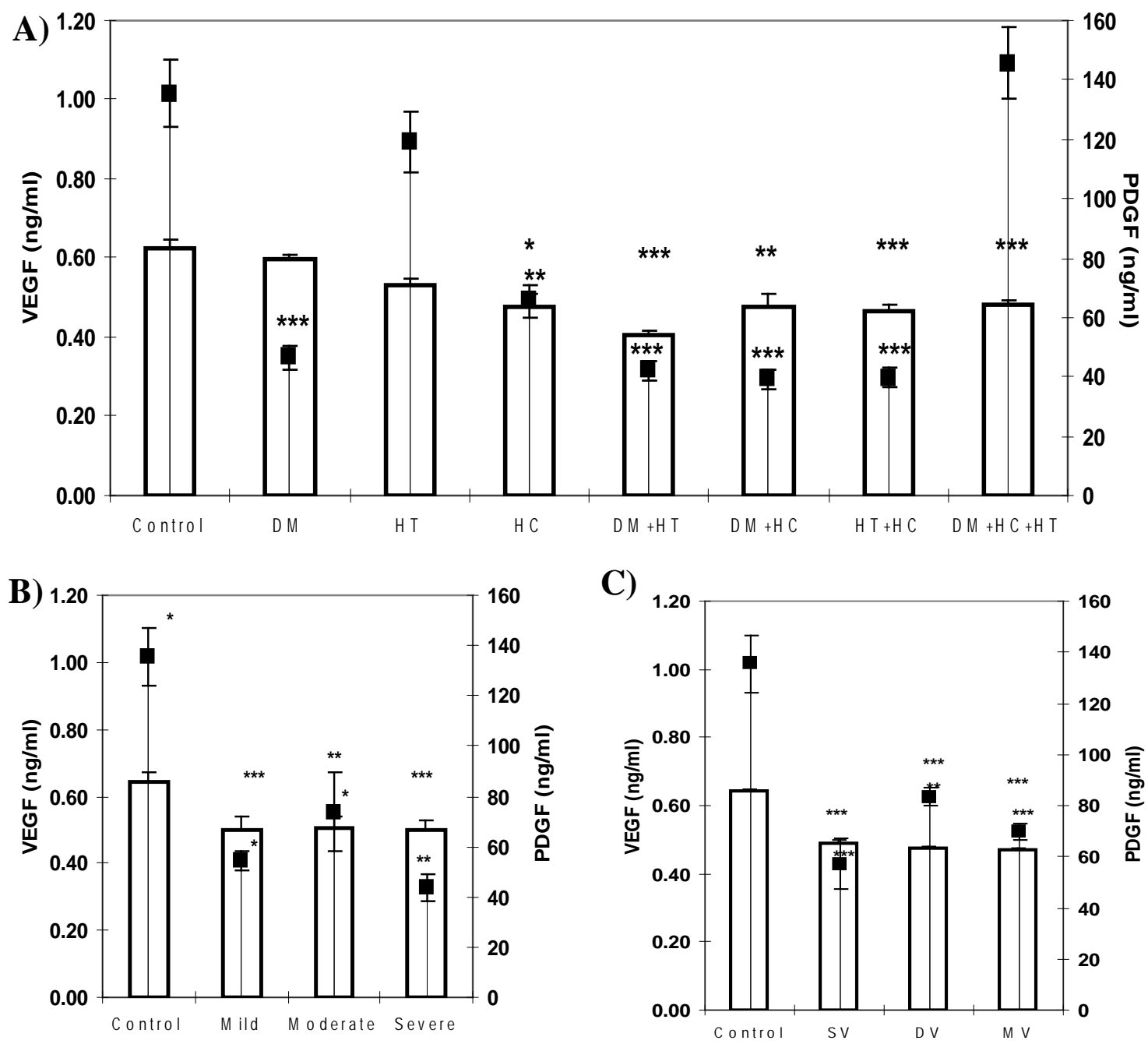

Figure 3: Plasma VEGF (white columns) and PDGF (black lines) in CAD patients classified according to risk factors (a), smoking status (b), and number of stenoic arteries (c). Results are expressed as mean \pm standard error. ${ }^{*}$ Denotes groups significantly different from controls ${ }^{*} P<0.05$, ${ }^{* *} P<0.01$, ***P $<0.001$.

DM: Diabetes Mellitus; HT: Hypertension; HC: Hypercholesterolemia; SV: Single Vessel Stenosis; DV: Double Vessel Stenosis; MV: Multi-Vessel Stenosis

$P<0.001)$ and cCD36 mRNA $(r=-0.328, P<=0.01)$. Statistically significant negative correlation was observed between TNF- $\alpha$ and VEGF production in plasma $(r=-0.323, P<0.01)$. sCD36 showed a significant positive correlation with cCD36 $(r=0.234, P$ $<0.05)$, and both of them showed a direct correlation with plasma $\operatorname{VEGF}(r=0.446, P<0.001$ and $r=0.321, P<0.01$; respectively), PDGF ( $r=0.287, P<0.05$ and $r=0.401, P<0.001$; respectively) and a negative correlation with plasma TNF- $\alpha(r=-0.352, P<0.01$ and $r=-0.380, P<0.001$; respectively).

Finally, using multiple linear regression, disease association remained significant $(\beta=-0.239, P<0.05)$ between serum levels of sCD36, taken as the dependent variable $\left(R^{2}=0.082\right)$, for the presence of down regulated TNF- $\alpha$. On the other hand, other independent variables (VEGF, PDGF) were not significant in the multivariate analysis.

\section{Discussion}

Atherosclerotic CAD progression, whether clinically silent or associated with acute coronary events, has been shown to be a powerful predictor of cardiovascular risk [17]. It is now widely accepted that atherosclerosis is a chronic inflammatory disease [18] and that CD36 as a scavenger receptor for oxidized LDL played an important role in the pathogenesis of atherosclerosis [19]. This study focused on the role of CD36 in the development of atherosclerotic CAD in Egyptian patients and the influence of various risk factors on CD36, with particular attention to its association with inflammatory and angiogenic mediators.

The findings showed that Egyptian CAD patients had reduced serum sCD36 levels compared to healthy controls. This observation indicated that sCD36 levels might be affected 


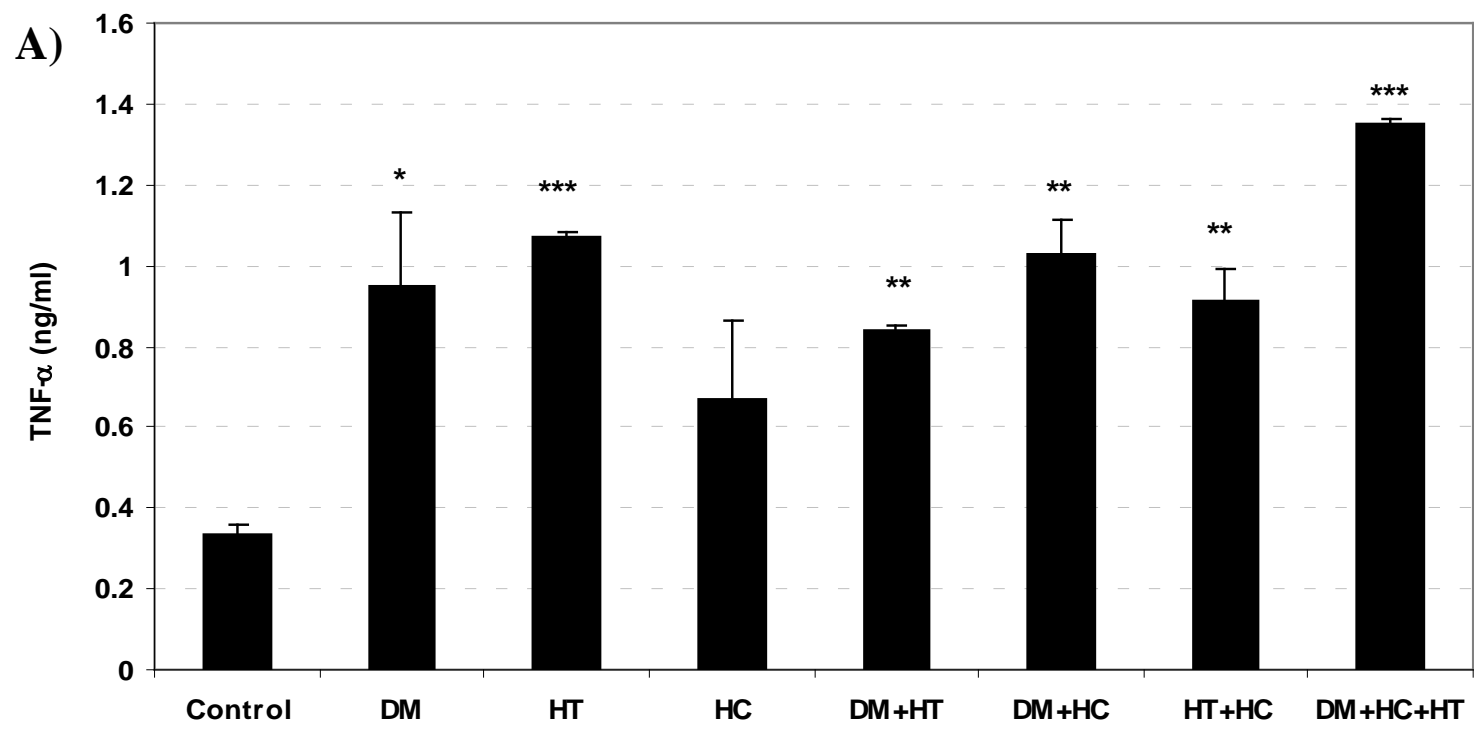

B)

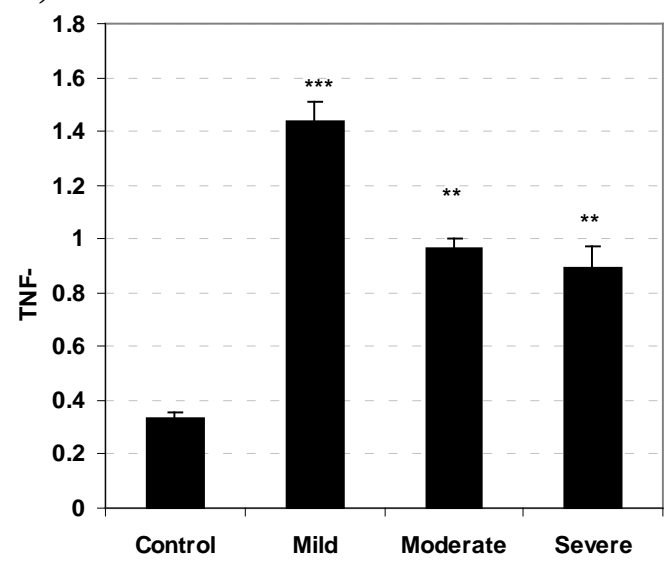

\section{C)}

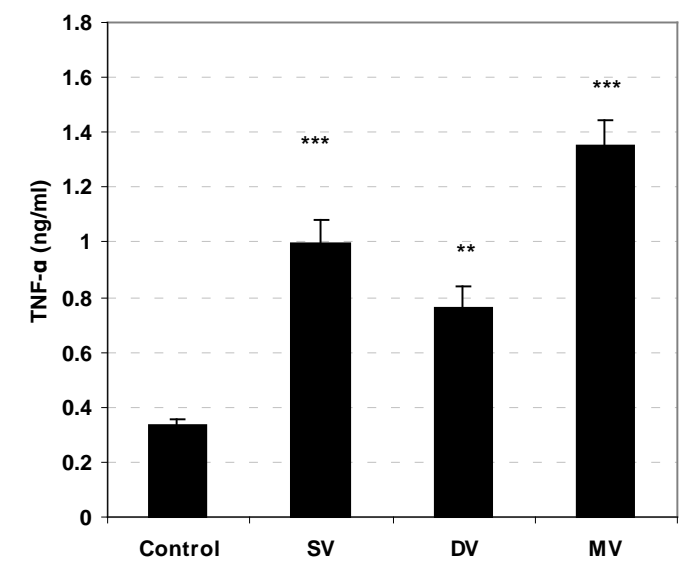

Figure 4: Plasma TNF- $\alpha$ in CAD patients classified according to risk factors (a), smoking status (b), and number of stenoic arteries (c). Results are expressed as mean \pm standard error. ( ${ }^{*}$ )Denotes groups significantly different from controls. ${ }^{*} P<0.05,{ }^{* *} P<0.01,{ }^{* * *} P<0.001$.

DM: Diabetes Mellitus; HT: Hypertension; HC: Hypercholesterolemia; SV: Single Vessel Stenosis; DV: Double Vessel Stenosis; MV: Multi-Vessel Stenosis

by the degree of smoking status in CAD patients and indicated that a reduction in plasma level of sCD36 could be considered as a potential marker and a strong predictor of CAD. These data are in agreement with previously reported data that suggested that sCD36 may be derived from a proteolytic cleavage of the extracellular part of CD36 protein from CD36- expressing tissues or from micro particles of activated or apoptotic monocytes/ macrophages [20]. A previous explanatory studies provided that soluble form of CD36 could serve as a biomarker that is associated with altered PBMCs CD36 expression, thus, the levels of sCD36 seem to be parallel CD36 expression on intact monocytes. Based on this study, the down regulated CD36 in our patients' plasma might be used as an indicator to a PBMCs CD36 down regulation. This hypothesis is confirmed through the detection of CD36 either in PBMCs surface and mRNA expression. A comparable remarkable result was observed, where a significant reduction in cCD36 expression was documented in our work in all types of risk factors.

Our data confirmed the previously reported studies [21-22]; that revealed the association of CD36 deficiency with HC, DM and arterial HT, and agreed with [23] who reported a severe atherosclerosis in subjects whom naturally deficient in CD36, which suggests that CD36 has an anti-atherogenic role. Elevation in LDL-Cholesterol was previously documented [24], modified LDL, and triglycerides [25] levels in CD36 deficient patients, which were considered as risk factors for CAD.

Furthermore, patients with severe stenosis (DV and MV) showed a marked reduction in CD36 mRNA than those with SV. These data suggest that CD36 mRNA expression is associated with the severity of CAD. However, the molecular mechanism by which the reduction of CD36 RNA expression occurs in PMMCs 
is still unclear. One possible explanation is that the decrease in CD36 mRNA might be linked to a mutation in CD36 gene that affects transcriptional efficiency in macrophages. A significant indirect correlation between CAD and CD36 profile (sCD36, cCD36 and CD36 mRNA) was found. Thus CD36 may turn out to be a good expression marker for the severity of atherosclerosis in Egyptian CAD patients.

CD36 not only implicated in atherosclerosis, but also has an angiostatic function, as demonstrated by the inability of thrombospondin-1 (TSP-1) to inhibit angiogenesis in CD36 null mice [26]. In atherosclerosis, the role of angiogenesis remains a highly contentious issue. No consensus exists whether angiogenesis is a key causative factor in the pathogenesis of atherosclerotic plaque formation or it is a way to treat coronary artery disease [27]. For further investigation of this issue, we extended our work to study the exact relationship between CAD and both of CD36 and angiogenic mediators in CAD patients.

Growth factors, such as VEGF and PDGF play an important role in angiogenesis. Dysfunction of endothelial cells may promote abnormal vascular growth, such as that in atherosclerosis and arteriosclerosis [28]. In the current study, we demonstrated that plasma VEGF was reduced markedly in all HC CAD groups. In addition, decreased plasma VEGF level was also found in DM CAD patients. These findings are consistence with previous work that reported a reduction in VEGF in diabetic rats, that followed by ischemic cardiovascular disease [29]. Our results strengthen previous reports that document the reduction in VEGF in diabetic rats, that followed by ischemic cardiovascular disease [30]. In agreement with the presented results, it was demonstrated that the reduction of VEGF levels lead to elevation of peripheral resistance and HT [31].

When considering smoking status, a marked reduction of VEGF and PDGF levels were observed in all groups. These results are in agreement with previous studies that reported a reduced level of VEGF as a result of cigarette smoking [31-32]. The observed reduction of VEGF among smokers of CAD patients might be related to the fact that cigarette smoke disrupts components of the VEGF165-VEGFR-2 tertiary signaling complex by decreasing neuropilin-1 (NRP-1) expression together with reducing expression of VEGFR-2 and VEGF [33]. Whereas, the current data demonstrated a significant decrease of VEGF and PDGF levels in plasma of CAD patients with SV, DVs, and MVs. A significant indirect correlation between CAD and angiogenic factors (VEGF and PDGF) is best expressed by the Pearson correlation coefficient analysis indicated that the VEGF has a prognostic importance in atherosclerotic CAD in Egyptian patients. Decreased VEGF and PDGF levels in CAD patients may be contributed to the reduction of CD36 expression. This explanation is supported by a direct correlation between CD36 and angiogenic mediators observed in our study. This is consistent with Howell, et al. [34] who reported a diminished expression VEGF in CD36 deficient mice.

Inflammatory mediators can; either directly or indirectly, promote angiogenesis, which in turn contributes to inflammatory pathology. New blood vessels can maintain the chronic inflammatory state by transporting inflammatory cells to the site of inflammation and supplying nutrients and oxygen to the proliferating inflamed tissue. The increased endothelial surface area also creates an enormous capacity for the production of cytokines, adhesion molecules, and other inflammatory stimuli. Generally speaking, pro-inflammatory mediators promote angiogenesis and the proangiogenic effects mediated by IL-1 and TNF- $\alpha$ support such hypothesis [35]. The consequences of inflammation in atherosclerosis are difficult to predict. Although it may be beneficial at early stage, with prosperities reversing atherogenesis, it may be detrimental to the individual with more aggressive disease progression and plaque rupture at later stage.

Mediators of inflammation such as TNF- $\alpha$ have been associated with an increased risk for cardiovascular events in several clinical studies [36]. TNF- $\alpha$ is a central pro-inflammatory cytokine involved in the propagation of atherosclerosis. TNF- $\alpha$ is secreted in the vascular wall by endothelial smooth muscle cells and monocytes/macrophages and it is a powerful inducer of local inflammation [37]. It promotes the expression of leukocyte adhesion molecules [38] and increases the uptake of macrophages in atherosclerotic lesions [39] thus directly promoting atherosclerosis.

The current study showed a significant elevation in TNF- $\alpha$ level in DM, HT and multi-risk factors CAD patients. In addition, a marked elevation in TNF- $\alpha$, plasma levels were reported in mild, moderate, and severe smokers, while the elevation is more significant in severe smokers than other groups. These results are in agreement with an earlier report that recorded elevated TNF- $\alpha$ in congestive heart failure that was associated with atherosclerotic risk factors, including smoking, hyperglycemia, HT, LDL cholesterol [40].

Inflammation plays a pivotal role in the development of metabolic syndrome features, including dyslipidemia and altered glucose tolerance. These metabolic changes constitute the substrate for the subsequent development of atherosclerotic plaque [41]. Chronic inflammatory conditions have been shown to be associated with pro-atherogenic lipid pattern and altered glucose tolerance. TNF- $\alpha$ has been demonstrated to directly interfere with metabolic pathways of triacylglyceride and cholesterol [42].

In addition to the modifications thatoccur in lipids metabolism, TNF- $\alpha$ may interfere with glucose metabolism pathways [43], where it is likely to increase hepatic glucose production and decreases glucose uptake and catabolism in the muscle. While, in adipocytes, TNF- $\alpha$ down regulate the expression of several proteins implicated in the insulin receptor pathway [44]. The lipid and glucose changes induced by TNF- $\alpha$ are pro-atherogenic in terms of both quality and quantity. Therefore, the persistence of these modified lipids in the circulation will promote the development of atherosclerotic lesions [41]. These data strongly support the hypothesis that the inflammatory cytokines (such as TNF- $\alpha$ ) are surrogate biomarker of grade inflammation burden present in patients with atherosclerotic CAD who suffered from DM or HT or both of them [45]. According to the number of 
stenotic coronary vessels, we reported an elevation in plasma TNF- $\alpha$ in SV, DVs, and MVs CAD patients, where TNF- $\alpha$ is more significantly elevated in MVs group than others. These data are supported by statistical analysis that showed a direct correlation between CAD and TNF- $\alpha$ level, which indicates the pivotal role played by TNF- $\alpha$ in atherosclerosis. In accordance with our data, it was previously found that the elevated plasma TNF- $\alpha$ concentration is associated with severity of atherosclerosis [46].

With regard to angiogenesis, our study showed an indirect correlation between TNF- $\alpha$ with VEGF and PDGF. Consistent with our data, it was reported that TNF- $\alpha$ directly inhibits VEGF cellular effects and that TNF-mediated inhibition of angiogenesis results from down regulation of receptors for proangiogenic factors and activation of angiogenesis inhibitors [47]. Furthermore, CD36 showed indirect correlation with TNF- $\alpha$ in CAD patients, which agreed with Boyer, et al. [48], who reported that TNF- $\alpha$ inhibits both CD36 membrane and mRNA expression and that this inhibition of CD36 expression involves a reduction in Peroxisome proliferator-activated receptor gamma (PPAR- $\gamma$ ) activation in human monocytes.

In conclusion, we found significant reduction of sCD36 and PBMCs CD36 surface protein and CD36 mRNA expression, for the first time in Egyptian CAD patients. CD36 was correlated with the severity of the disease. Our data approved that CD36 deficiency may turn out to be a good biomarker and strong predictor for the severity of atherosclerosis in Egyptian CAD patients. Furthermore, the marked elevation of inflammatory cytokine TNF- $\alpha$ support the hypothesis that TNF- $\alpha$ could be used as a surrogate marker of grade inflammation present in patients with atherosclerosis and a pivotal role of TNF- $\alpha$ in elevation of atherosclerosis. On the other hand, the marked reduction in proangiogenic mediators (VEGF \& PDGF) and the strong indirect correlation between VEGF and CAD indicating that VEGF has a prognostic importance in atherosclerosis in CAD patients.

Taken together CD36 deficiency might represent a new risk factor for the development of CAD in association with inflammatory and angiogenic mediators. Therefore, targeting the underlying mechanism of the direct and inverse association of CD36 with angiogenic mediators (VEGF \& PDGF) and inflammatory cytokine (TNF- $\alpha$ ) in Egyptian CAD patients could be a strategy in the treatment of athero-inflammatory disorders and deserves exploration in future prospective study. Our assessment of this evidence leads us to conclude that, there is a need for early-stage prediction of those populations that have the risk of developing atherosclerotic CAD. This need could be met by analysis of CD36 genetic polymorphisms related to CD36 deficiency. Thus, our future prospective study will be directed towards studying the association between change in CD36 genotype and the incidence of CAD in a large, prospective cohort of Egyptian patients

\section{Acknowledgement}

This work was financially supported by the National Research Center, Cairo, Egypt.

\section{Conflict of Interest}

We declare no conflict of interest.

\section{Author Contribution}

Enas, Amira, Nadia and Mohamed participated in the experimental design. Enas and Roba conducted the experiments. Enas, Amira and Roba interrelated and analyzed the data. Ahmed Tamara participated in clinical work. All Authors participated in writing the manuscript and approved its final version.

\section{References}

1. Wang Q. Molecular genetics of coronary artery disease. Curr Opin Cardiol. 2005; 20(3): 182-188.

2. World Health Organization (WHO): The annual health report of the year 2009. Cairo, WHO, 2009.

3. Central Agency for Public Mobilization and Statistics (CAPMAS): The annual health report of the year 1990. Cairo, CAPMAS, 1990.

4. Wilson J M. Diagnosis and treatment of acquired coronary artery disease in adults. Postgrad Med J. 2009; 85(1005): 364-365. doi: 10.1136/pgmj.2008.068791.

5. Libby P. Inflammation and cardiovascular disease mechanisms. Am J Clin Nutr. 2006; 83(2): 456S-460S

6. Ouguerram K, Nguyen P, Krempf M, Pouteau E, Briand F, Bailhache E, et al. Selective uptake of high-density lipoproteins cholesteryl ester in the dog, a species lacking in cholesteryl ester transfer protein activity; an in vivo approach using stable isotopes. Comp Biochem Physiol B Biochem Mol Biol. 2004; 138(4): 339-345.

7. Abd Allah F, Baligh E, Ibrahim M. Clinical relevance of carotid atherosclerosis among Egyptians: a 5-year retrospective analysis of 4,733 subjects. Neuroepidemiology. 2010; 35(4): 275-279. doi: $10.1159 / 000319899$

8. Yamashita S, Hirano K, Kuwasako T, Janabi M, Toyama Y, Ishigami M, et al. Physiological and pathological roles of a multi-ligand receptor CD36 in atherogenesis; insights from CD36-deficient patients. Mol Cell Biochem. 2007; 299(1-2): 19-22.

9. Collot-Teixeira S, Martin J, McDermott-Roe C, Poston R, McGregor JL. CD36 and macrophages in atherosclerosis. Cardiovasc Res. 2007; 1; 75(3): 468-477

10. Febbraio M, Podrez EA, Smith JD, Hajjar DP, Hazen SL, Hoff HF, et al. Targeted disruption of the class B scavenger receptor CD36 protects against atherosclerotic lesion development in mice. J Clin Invest. 2000; 105(8): 1049-1056

11. Connolly DT, Heuvelman DM, Nelson R, Olander JV, Eppley BL, Delfino JJ, et al. Tumor vascular permeability factor stimulates endothelial cell growth and angiogenesis. J Clin Invest. 1989; 84(5): 1470-1478.

12. Uchida K. A Lipid-derived Endogenous Inducer of COX-2: a Bridge between Inflammation and Oxidative Stress. Mol Cells. 2008; 25(3): 347-351.

13. Bigda J, Okrój M, Onkol W. The role of tumor necrosis factor (TNF) in angiogenesis. Often has. 2002; 6(2): 57-59.

14. FitzGerald GA. COX-2 and beyond: approaches to prostaglandin inhibition in human disease. Nat Rev Drug Discov. 2003; 2(11): 879 890

15. Bassyouni IH, Gheita TA, Talaat RM. Clinical significance of serum levels of sCD36 in patients with systemic sclerosis: preliminary data. Rheumatology (Oxford). 2011; 50(11): 2108-2112. doi: 10.1093/ rheumatology/ker297.

16. Talaat RM. Soluble angiogenesis factors in sera of Egyptian patients 
with hepatitis $C$ virus infection: correlation with disease severity Viral Immunol. 2010; 23(2): 151-157. doi: 10.1089/vim.2009.0089.

17. Ross R. Atherosclerosis-an inflammatory disease. N Engl J Med. 1999; 14; 340(2): 115-126.

18. Tiong A Y, Brieger D. Inflammation and coronary artery disease. Am Heart J. 2005; 150(1): 11-18.

19. Handberg A, Levin K, Hojlund K, Beck- Nielsen H. Identification of the oxidized low-density lipoprotein scavenger receptor CD36 in plasma: a novel marker of insulin resistance. Circulation. 2006; 12; 114(11): 1169-1176. Epub 2006 Sep 4.

20.Wang L, Bao Y, Yang Y, Wu Y, Chen X, Si S, et al. Discovery of antagonists for human scavenger receptor CD36 via an ELISA like high-throughput screening assay. J Biomol Screen. 2010; 15(3): 239250. doi: $10.1177 / 1087057109359686$.

21. Hajri T, Han XX, Bonen A, Abumrad NA. Defective fatty acid uptake modulates insulin responsiveness and metabolic responses to diet in CD36-null mice. J Clin Invest 2002, 109(10):138-1389.

22. Miyaoka K, Kuwasako T, Hirano K, Nozaki S, Yamashita S, Matsuzawa Y. CD36 deficiency associated with insulin resistance. Lancet. 2001; 357(9257):686-687.

23. Masuda D, Hirano K, Oku H, Sandoval JC, Kawase R, Yuasa-Kawase M, et al. Chylomicron Remnants Are Increased in the Postprandial State in CD36 Deficiency. J Lipid Res. 2009; 50: 999-1011. doi: 10.1194/jlr. P700032-JLR200.

24. Sato 0, Kuriki C, Fukui Y, Motojima K. Dual promoter structure of mouse and human fatty acid translocase/CD36 genes and unique transcriptional activation by peroxisome proliferator-activated receptor alpha and gamma ligands. J Biol Chem. 2002; 277: 1570315711.

25. Khurana R, Simons M, Martin JF, Zachary IC. Role of Angiogenesis in Cardiovascular Disease: A Critical Appraisal. Circulation. 2005; 112(12):1813-1824.

26. Dzau VJ. The role of mechanical and humoral factors in growth regulation of vascular smooth muscle cells and cardiac myocytes. Curr Opin Nephrol Hypertens. 1993;2(1): 27-32.

27.Yoon YS, Uchida S, Masuo O, Cejna M, Park JS, Gwon HC, et al. Progressive Attenuation of Myocardial Vascular Endothelial Growth Factor Expression Is a Seminal Event in Diabetic Cardiomyopathy. Circulation. 2005; 111(16): 2073-2085.

28. Koyama S, Sato E, Haniuda M, Numanami H, Nagai S, Izumi T. Decreased level of vascular endothelial growth factor in bronchoalveolar lavage fluid of normal smokers and patients with pulmonary fibrosis. Am J Respir Crit Care Med. 2002; 166(3): 382-385.

29. Khorram O, Khorram N, Momeni M, Han G, Halem J, Desai M, et al. Maternal undernutrition inhibits angiogenesis in the offspring: a potential mechanism of programmed hypertension. Am J Physiol Regul Integr Comp Physiol. 2007; 293(2): R745-R753.

30. Felmeden DC, Spencer CGC, Chung NA, Belgore FM, Blann AD, Beevers DG, et al. Relation of thrombogenesis in systemic hypertension to angiogenesis and endothelial damage/ dysfunction (a Sub study of the Anglo-Scandinavian Cardiac Outcomes Trial [ASCOT]. Am J Cardiol. 2003; 92: 400-405.

31. Melkonian G, Cheung L, Marr R, Tong C, Talbot P. Mainstream and Sidestream Cigarette Smoke Inhibit Growth and Angiogenesis in the Day 5 Chick Chorioallantoic Membrane. Toxicolo Sci. 2002; 68: 237 248.
32. Cheng SL, Wang HC, Yu CJ, Yang PC. Increased expression of placenta growth factor in COPD. Thorax. 2008; 63: 500-506. doi: 10.1136/ thx.2007.087155.

33. Houssier M, Raoul W, Lavalette S, Keller N, Guillonneau X, Baragatti B, et al. CD36 Deficiency Leads To Choroidal Involution Via COX2 DownRegulation In Rodents. PLos Med. 2008; 5(2): E39. doi: 10.1371/ journal.pmed.0050039.

34. Howell WM, Ali S, Rose-Zerilli MJ, Ye S. VEGF polymorphisms and severity of atherosclerosis. J Med Genet. 2005; 42(6): 485-490.

35. Leung, DW, Cachianes G, Kuang WJ, Goeddel DV, Ferrara N. Vascular endothelial growth factor is a secreted angiogenic mitogen. Science. 1989; 246(4935): 1306-1309.

36. Ridker PM, Rifai N, Pfeffer M, Sacks F, Lepage S, Braunwald E. Elevation of tumor necrosis factor-and increased risk of recurrent coronary events after myocardial infarction. Circulation. 2000; 101(18): 21492153.

37. Libby P. Inflammation in atherosclerosis. Nature. 2002; 420(6917): 868-874.

38. Kim CJ, Khoo JC, Gillotte-Taylor K, Li A, Palinski W, Glass CK, et al. Polymerase chain reaction-based method for quantifying recruitment of monocytes to mouse atherosclerotic lesions in vivo: enhancement by tumor necrosis factor-alpha and interleukin-1 beta. Arterioscler Thromb Vasc Biol. 2000; 20(8): 1976-82.

39. Skoog T, Dichtl W, Boquist S, Skoglund-Andersson C, Karpe F, Tang R et al. Plasma tumor necrosis factor-alpha and early carotid atherosclerosis in healthy middle-aged men. Eur Heart J. 2002; 23(5):376-383.

40. Popa C, Netea MG, van Riel PL, van der Meer J W, Stalenhoef AF. The role of TNF- $\alpha$ in chronic inflammatory conditions, intermediary metabolism, and cardiovascular risk. J Lipid Res. 2007; 48(4): 751762.

41. Feingold KR, Pollock AS, Moser AH, Shigenaga JK, Grunfeld C. Discordant regulation of proteins of cholesterol metabolism during the acute phase response. J Lipid Res. 1995; 36(7): 1474-1482.

42. Memon RA, Grunfeld C, Moser AH, Feingold KR. Tumor necrosis factor mediates the effects of endotoxin on cholesterol and triglyceride metabolism in mice. Endocrinology. 1993; 132(5): 2246-2253.

43. Feingold KR, Hardardottir I, Grunfeld C. Beneficial effects of cytokine induced hyperlipidemia. Z Ernahrungswiss. 1998; 37: 66-74.

44. Stephens JM, Pekala PH. Transcriptional repression of the GLUT4 and C/EBP genes in 3T3-L1 adipocytes by tumor necrosis factor-alpha. J Biol Chem. 1991; 266(32): 21839-21845.

45. Elneihoum AM, Falke P, Hedblad B, Lindgarde F, Ohlsson K. Leukocyte activation in atherosclerosis: correlation with risk factors. Atherosclerosis. 1997; 131(1): 79-84.

46. Gotsman I, Stabholz A, Planer D, Pugatsch T, Lapidus L, Novikov Y, et al. Serum Cytokine Tumor Necrosis Factor-Alpha and Interleukin 6 Associated with the severity of Coronary Artery Disease: Indicators of an Active Inflammatory Burden? Isr Med Assoc J. 2008; 10(7): 494498.

47. Nakagami H, Cui T-X, Iwai M, Shiuchi T, Takeda-Matsubara Y, Wu L, et al. Tumor Necrosis Factor- Inhibits Growth Factor-Mediated Cell Proliferation Through SHP-1 Activation in Endothelial Cells. Arterioscler Thromb Vasc Biol. 2002; 22(2): 238-242.

48. Boyer JF, Balard P, Authier H, Faucon B, Bernad J, Mazières B, et al. Tumor necrosis factor alpha and adalimumab differentially regulate CD36 expression in human monocytes. Arthritis Research \& Therapy. 2007; 9(2): R22. 